Review began 10/14/2021 Review ended 10/25/2021 Published 11/01/2021

๑) Copyright 2021

Kobayashi et al. This is an open access article distributed under the terms of the Creative Commons Attribution License CCBY 4.0., which permits unrestricted use, distribution, and reproduction in any medium, provided the original author and source are credited.

\section{Open Reduction and Internal Fixation of Simultaneous, Bilateral, Atypical Femoral Shaft Fractures After Nine Years of Bisphosphonate Treatment}

\author{
Tatsuki Kobayashi $^{1}$, Tetsuhiro Ishikawa ${ }^{1}$, Joe Katsuragi ${ }^{1}$, Yasuhito Sasaki ${ }^{1}$, Seiji Ohtori ${ }^{2}$ \\ 1. Orthopedic Surgery, Sanmu Medical Center, Chiba, JPN 2. Orthopedic Surgery, Graduate School of Medicine, Chiba
} University, Chiba, JPN

Corresponding author: Tatsuki Kobayashi, tatsuki.chocolat@icloud.com

\begin{abstract}
Bisphosphonates are generally used to treat osteoporosis and decrease the risk of femoral neck and vertebral fractures in patients with osteoporosis. Recently, it has been suggested that long-term bisphosphonate use can lead to decreased bone remodeling and an increased risk of atypical fractures. Atypical fractures often occur in the femur. The purpose of the present report is to describe a rare case of simultaneous, bilateral, atypical femoral fractures. An 80-year-old female was walking when she sustained bilateral femoral fractures that were diagnosed as atypical. The patient had received bisphosphonate treatment over the prior nine years.
\end{abstract}

Categories: Orthopedics

Keywords: autobiographical case report, open reduction and internal fixation, bilateral, simultaneous, bisphosphonate, atypical fracture

\section{Introduction}

Bisphosphonates are one of the most prescribed drugs for the treatment of osteoporosis because they decrease the risk of femoral neck and vertebral fractures in patients with osteoporosis [1,2]. Bisphosphonate therapy can increase bone marrow density (BMD), although the BMD response typically reaches a plateau in two to three years [3].

There is evidence to suggest that prolonged use of bisphosphonates can suppress bone remodeling and increase the risk of atypical fractures. For this reason, physicians can consider a drug holiday after long-term bisphosphonate use (three to 10 years) to reduce the risk of such complications [1]. Reportedly, several prodromal symptoms are likely reported before atypical femoral fractures (AFFs) occur and physicians need to be aware of them in the patients for whom they are prescribing antiresorptive drugs [2].

Atypical fractures occur frequently in the femoral subtrochanteric region or shaft [2]. Most reports of AFFs refer to unilateral cases. Here we describe a rare case of simultaneous, bilateral AFFs.

\section{Case Presentation}

An 80-year-old female fell when walking. At the moment of catastrophic injury, the patient described hearing a noise coming from both thighs before falling to the ground. A radiographic examination showed bilateral femoral shaft fractures (Figure 1). 


\section{Cureus}

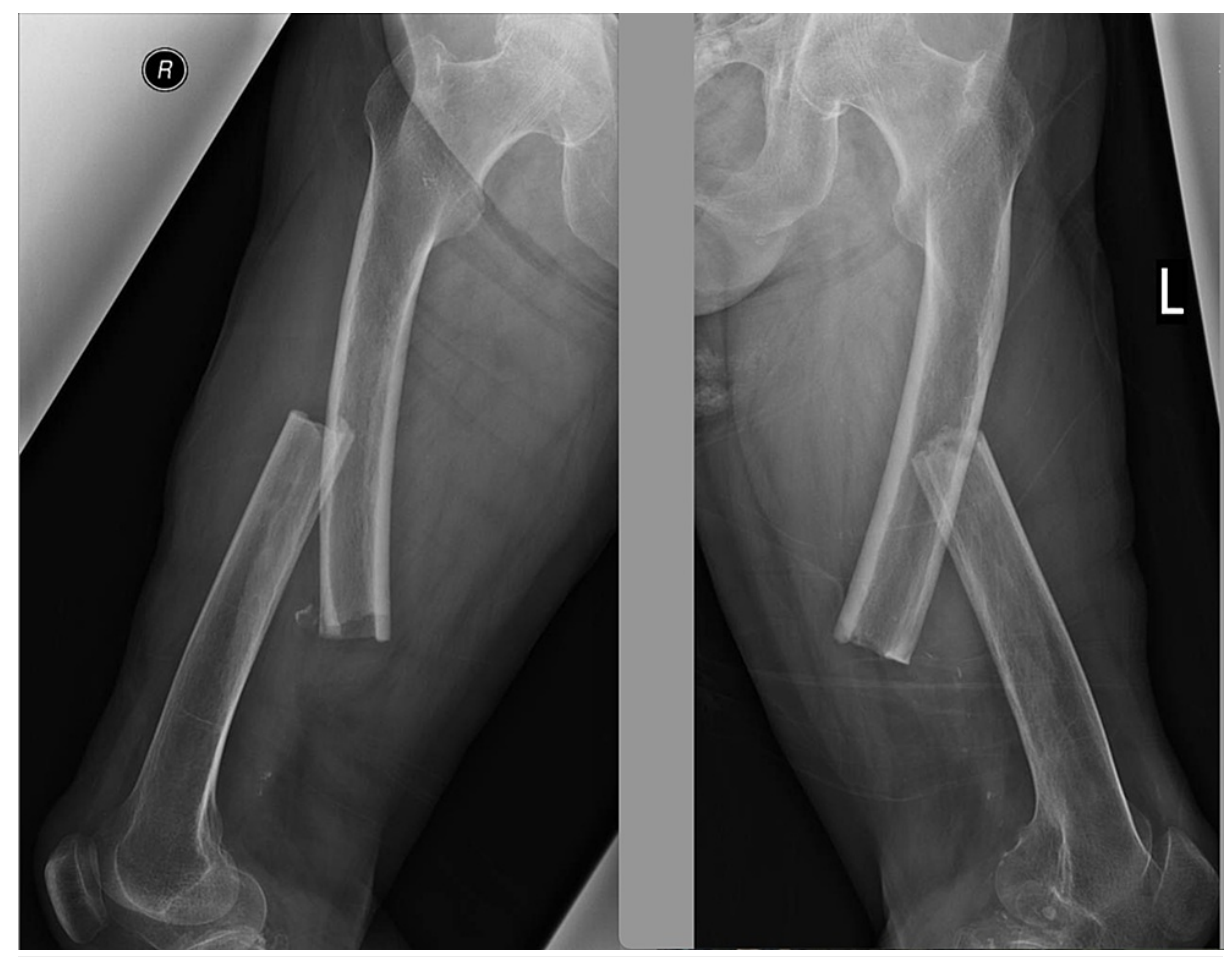

FIGURE 1: Plain radiographs of the right and left femurs.

Bilateral transverse fractures with cortical thickening suggest the fractures were atypical.

Her medical history revealed that she had been prescribed $2.5 \mathrm{mg} /$ day of risedronate for the prior nine years as a treatment for osteoporosis. Radiographs and computed tomographic (CT) images revealed a transverse fracture in the middle of each femur, and cortical thickening was evident at both fracture sites. A dualenergy X-ray absorptiometry scan showed that her lumbar vertebral BMD was $54 \%$ of that of adults aged 20 44 years. The patient's T-score in the spine was -3.7 and $\mathrm{Z}$-score was -0.1 . Blood tests indicated that her serum intact amino-terminal propeptide of type 1 procollagen was $128 \mu \mathrm{g} / \mathrm{L}$ (26.4-98.2) and her tartrateresistant acid phosphatase $5 \mathrm{~b}$ was $541 \mu \mathrm{U} / \mathrm{dL}(120-420)$. The patient was diagnosed with simultaneous bilateral AFFs. Open reduction and internal fixation of the fractures were performed using an intramedullary (IM) nail system (T2 Nail, Stryker Corp., Kalamazoo, MI) (Figure 2). 


\section{Cureus}

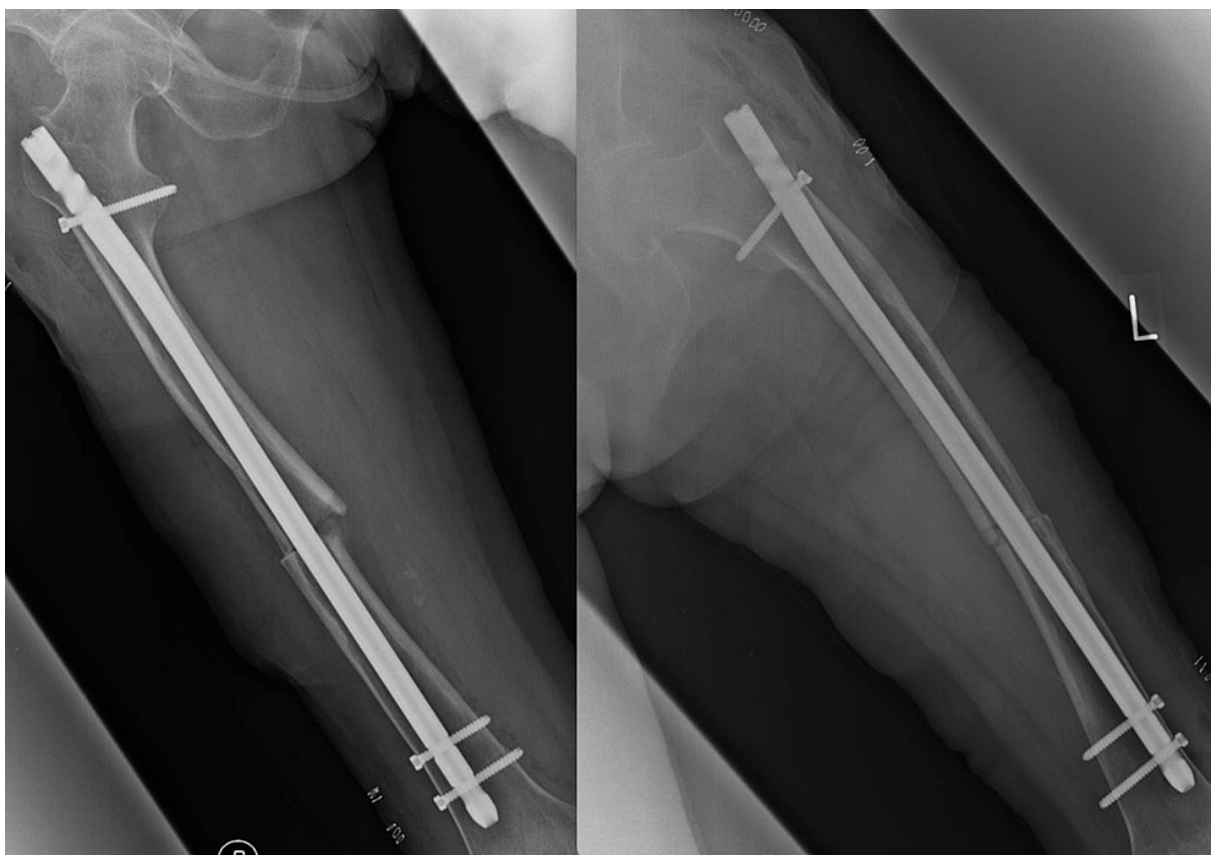

FIGURE 2: Plain radiographs of the right and left femurs immediately after surgery.

It was imperative for the patient to not bear weight on either leg for six weeks after surgery. During the four months of hospitalization, walking practice was included as part of the patient's rehabilitation until she was eventually discharged with a walking cane. Bone union was observed and eight months after her surgeries, she was able to walk without the assistance of a cane (Figure 3).

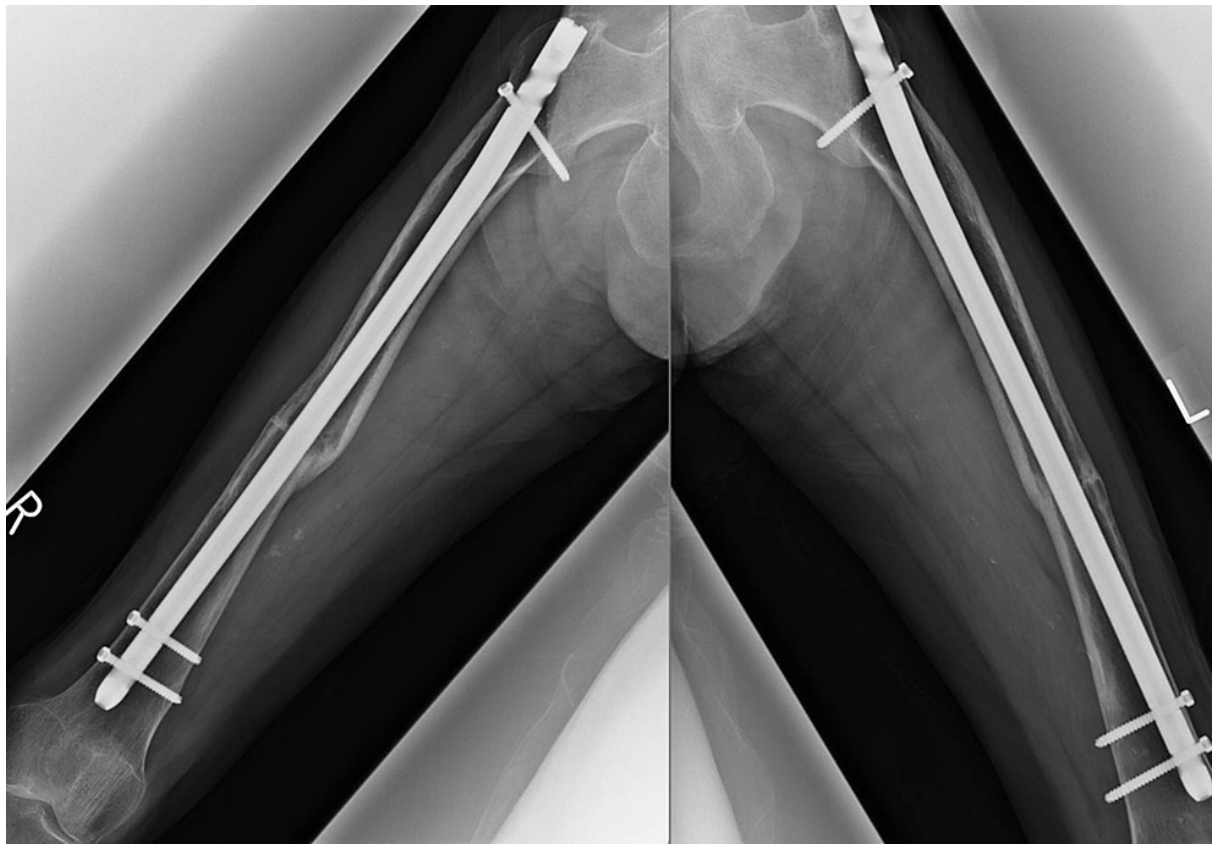

FIGURE 3: Plain radiographs of the right and left femurs one year after surgery when bone union was observed.

\section{Discussion}

The American Society of Bone and Mineral Research Task Force case definition of AFFs is shown in Table 1 [1]. This case report exhibited features similar to those typically observed as major features of AFFs, such as no associated trauma or minimal trauma after falling from a standing height or less. As shown in Table 1 , 


\section{Cureus}

other examples of major features are a transverse or short oblique configuration, noncomminuted, complete fractures extending through both cortices which may be associated with a medial spike, or incomplete fractures only involving the lateral cortex. Minor features of AFFs were also evident in this case; there was a generalized increase in shaft cortical thickness, bilateral fractures, and bilateral prodromal pain at the thighs.

\section{Major features ${ }^{a}$}

Located anywhere along the femur from just distal to the lesser trochanter to just proximal to the supracondylar flare

Associated with no trauma or minimal trauma, as in a fall from a standing height or less

Transverse or short oblique configuration

Noncomminuted

Complete fractures extend through both cortices and may be associated with a medial spike; incomplete fractures involve only the lateral cortex

Minor features

Localized periosteal reaction of the lateral cortex ${ }^{\mathrm{b}}$

Generalized increase in cortical thickness of the diaphysis

Prodromal symptoms such as dull or aching pain in the groin or thigh

Bilateral fractures and symptoms

Delayed healing

Comorbid conditions (e.g., vitamin D deficiency, rheumatoid arthritis, hypophosphatasia)

Use of pharmaceutical agents (e.g., BPs, glucocorticoids, proton pump inhibitors)

Specifically excluded are fractures of the femoral neck, intertrochanteric fractures with spiral subtrochanteric extension, pathological fractures associated with primary or metastatic bone tumors, and periprosthetic fractures.

\section{TABLE 1: The ASBMR Task Force case definition of AFFs.}

AFF: atypical femur fracture; ASBMR: American Society for Bone and Mineral Research; BP: bisphosphonate

aAll major features are required to satisfy the case definition of AFF. None of the minor features are required but have been sometimes associated with these fractures.

${ }^{b}$ Often referred to in the literature as beaking or flaring

Bisphosphonates are among the most prescribed drugs for the treatment of osteoporosis to reduce the risk of bone fractures. Bisphosphonates increase BMD, although the BMD response typically reaches a plateau in two to three years [3]. In contrast, in a systematic review of all case reports and case series of AFFs, ParkWyllie et al. reported a median time of five years of bisphosphonate therapy prior to fracture [4]. AFFs represent approximately $0.5-1 \%$ of proximal femoral fractures [2]. There are few case reports of simultaneous bilateral AFFs; only seven published reports were retrieved during our search (Table 2). The duration of bisphosphonate use was only reported in five of seven published cases [5-10]. Three of those five documented cases were reports of bisphosphonate use for more than five years (Table 2) [5-7]. In our case, the patient discontinued her bisphosphonate regimen and received (SR3 and SR4) $1 \mu \mathrm{g} /$ day of alfacalcidol and $800 \mathrm{mg} /$ day of calcium L-aspartate hydrate. 


\section{Cureus}

\begin{tabular}{|c|c|c|c|c|c|c|}
\hline $\begin{array}{l}\text { Reports of } \\
\text { cases }\end{array}$ & $\begin{array}{l}\text { Reference } \\
\text { number }\end{array}$ & Age/sex & Bisphosphonate & $\begin{array}{l}\text { Duration ( } \\
\text { years) }\end{array}$ & $\begin{array}{l}\text { Mechanism of } \\
\text { injury }\end{array}$ & $\begin{array}{l}\text { Prodromal } \\
\text { symptoms }\end{array}$ \\
\hline \multirow{2}{*}{ Kamijo et al. } & \multirow{2}{*}{ [5] } & $59 / F$ & Alendronate & 4 & Fall & $(-)$ \\
\hline & & $74 / F$ & Minodronic acid & 7 & Fall & $(+)$ \\
\hline Higgins et al. & [6] & $71 / F$ & Alendronate & 8 & Fall & $(+)$ \\
\hline Zafeiris et al. & [7] & 76/M & Alendronate & 11 & Fall & $(+)$ \\
\hline Puah and Tan & [8] & $64 / \mathrm{M}$ & Alendronate & 1 & Fall & $(+)$ \\
\hline Ninomiya et al. & [9] & $80 / F$ & - & - & - & - \\
\hline Ovaska et al. & [10] & $60 / \mathrm{F}$ & Risedronate & - & - & - \\
\hline
\end{tabular}

TABLE 2: Past reports of cases of simultaneous bilateral AFF.

AFF = atypical femur fracture

For several weeks or months before an AFF occurs, approximately $70 \%$ of patients present with groin or thigh pain [1]. For patients who have been prescribed antiresorptive drugs (e.g., bisphosphonates or denosumab) and report such symptoms, physicians should consider performing full-length radiographs of both femurs [11]. Githens et al. suggested that if the patient is asymptomatic but presents with radiographic signs that raise concern about an impending atypical fracture, prophylactic IM nailing is recommended [12]. It has been shown that administration for teriparatide treatment in patients with an atypical fracture may help facilitate fracture healing, recovery of hip function, and pain relief when bone turnover is reduced [13].

\section{Conclusions}

In summary, this case report describes AFFs that occurred bilaterally and concurrently. The fractures were associated with long-term bisphosphonate use. Open reduction and internal fixation of the fractures were performed using an IM nail system. Bone union was achieved, and the patient was eventually able to walk again without assistance. Typically, AFFs occur unilaterally, although in rare cases, they can occur bilaterally. Physicians need to be aware of the effects of prescribing bisphosphonates for prolonged periods and need to consider a drug holiday after long-term bisphosphonate therapy to reduce the risk of atypical fractures. Another important point is to not overlook the prodromal symptoms of patients.

\section{Additional Information \\ Disclosures}

Human subjects: Consent was obtained or waived by all participants in this study. Conflicts of interest: In compliance with the ICMJE uniform disclosure form, all authors declare the following: Payment/services info: All authors have declared that no financial support was received from any organization for the submitted work. Financial relationships: All authors have declared that they have no financial relationships at present or within the previous three years with any organizations that might have an interest in the submitted work. Other relationships: All authors have declared that there are no other relationships or activities that could appear to have influenced the submitted work.

\section{References}

1. Shane E, Burr D, Abrahamsen B, et al.: Atypical subtrochanteric and diaphyseal femoral fractures: second report of a task force of the American Society for Bone and Mineral Research. J Bone Miner Res. 2014, 29:123. 10.1002/jbmr.1998

2. Feldstein AC, Black D, Perrin N, et al.: Incidence and demography of femur fractures with and without atypical features. J Bone Miner Res. 2012, 27:977-86. 10.1002/jbmr.1550

3. Anastasilakis AD, Polyzos SA, Makras P: Therapy of endocrine disease: denosumab vs bisphosphonates for the treatment of postmenopausal osteoporosis. Eur J Endocrinol. 2018, 179:31-45. 10.1530/EJE-18-0056

4. Park-Wyllie LY, Mamdani MM, Juurlink DN, et al.: Bisphosphonate use and the risk of subtrochanteric or femoral shaft fractures in older women. JAMA. 2011, 305:783-9. 10.1001/jama.2011.190

5. Kamijo H, Oniduka T, Izumi T, Omori Y, Watanabe E: Bilateral atypical femoral fracture: three cases reports. [Artcle in Japanese]. Orthop Traumatol. 2015, 64:411-3.

6. Higgins M, Morgan-John S, Badhe S: Simultaneous, bilateral, complete atypical femoral fractures after longterm alendronate use. J Orthop. 2016, 13:401-3. 10.1016/j.jor.2016.07.007

7. Zafeiris CP, Stathopoulos IP, Kourkoumelis G, Gkikas E, Lyritis GP: Simultaneous bilateral atypical femoral fractures after alendronate therapy. J Musculoskelet Neuronal Interact. 2012, 12:262-4.

8. Puah KL, Tan MH: Bisphosphonate-associated atypical fracture of the femur: spontaneous healing with 


\section{Cureus}

drug holiday and re-appearance after resumed drug therapy with bilateral simultaneous displaced fractures-a case report. Acta Orthop. 2011, 82:380-2. 10.3109/17453674.2011.581267

9. Ninomiya N, Matsumoto H, Kubota Y: Case report of both of femoral diaphysis pathological fractures in follow-up pubic bone fracture. [Artcle in Japanese]. J Japan Soc Fracture Repair. 2018, 287:494.

10. Ovaska MT, Mäkinen TJ, Madanat R: Simultaneous bilateral subtrochanteric fractures following risedronate therapy. J Orthop Sci. 2011, 16:467-70. 10.1007/s00776-011-0058-y

11. Khan AA, Kaiser S: Atypical femoral fracture. CMAJ. 2017, 189:542. 10.1503/cmaj.160450

12. Githens M, Garner MR, Firoozabadi R: Surgical management of atypical femur fractures associated with bisphosphonate therapy. J Am Acad Orthop Surg. 2018, 26:864-71. 10.5435/JAAOS-D-16-00717

13. Yeh WL, Su CY, Chang CW, Chen CH, Fu TS, Chen LH, Lin TY: Surgical outcome of atypical subtrochanteric and femoral fracture related to bisphosphonates use in osteoporotic patients with or without teriparatide treatment. BMC Musculoskelet Disord. 2017, 18:10.1186/s12891-017-1878-5 